\title{
PREVALENCE OF OBSTRUCTIVE SLEEP APNOEA IN ODISHA POPULATION- A CAMP BASED QUESTIONNAIRE SURVEY
}

\author{
Snigdha Pattanaik ${ }^{1}$, R. Rajagopal2, Neeta Mohanty33, Pragyna Panigrahi' ${ }^{4}$, Subhrajeet Narayan Sahoo 5
}

${ }^{1}$ Associate Professor, Department of Orthodontics and Dentofacial Orthopaedics, Institute of Dental Sciences, Siksha 'O'Anusandhan (Deemed to be University), Bhubaneswar, Odisha, Ph.D Scholar, Saveetha Institute of Medical and Technical Sciences, Thandalam, Chennai, India.

2Professor, Department of Orthodontics and Dentofacial Orthopaedics, Saveetha Dental College, Saveetha Institute of Medical and Technical Sciences, Thandalam, Chennai, India.

3Professor, Department of Oral and Maxillofacial Pathology and Oral Microbiology, Institute of Dental Sciences, Siksha 'O' Anusandhan (Deemed to be University), Bhubaneswar, Odisha, India.

${ }^{4}$ Postgraduate Student, Department of Orthodontics and Dentofacial Orthopaedics, Institute of Dental Sciences, Siksha 'O' Anusandhan (Deemed to be University), Bhubaneswar, Odisha, India.

${ }^{5}$ Assistant Professor, Department of Orthodontics and Dentofacial Orthopaedics, Institute of Dental Sciences, Siksha 'O' Anusandhan (Deemed to be University), Bhubaneswar, Odisha, India.

\section{BACKGROUND}

ABSTRACT

Obstructive sleep apnoea (OSA) is a disorder of most serious form among the sleep disordered breathing, which has become highly prevalent and negatively affects the quality of life. Its prevalence globally is reported to be $\geq 4 \%$ of men and $\geq 2 \%$ of women and affects mostly the obese individuals. Asymptomatic OSA has also been reported to be independently associated with an increased morbidity and mortality. Hence, it becomes a prime concern for health care personnel to diagnose it at the earliest. Among the various screening tools available to diagnose the Epworth Sleepiness Scale (ESS), it has been proved to be most accurate. Thus, this study was taken up to assess the prevalence of OSA in Odisha population using Epworth Sleepiness Scale.

\section{MATERIALS AND METHODS}

A total no. of 1012 subjects were selected using random sampling technique from various community health camps for the study. The subjects were asked to fill in the questionnaire for Epworth Sleepiness Scale. All questionnaire respondents were precisely briefed about this study in a face-to-face interview. The data obtained from the survey was subjected to statistics and descriptive analysis was done.

\section{RESULTS}

ESS score was found to be the highest in the age group above 70 (18.8\%) followed by age group 50 - 59 (17.7\%). ESS in 40 - 49 years' age group was $13 \%$ and $11.4 \%$ for the age group 40 - 49 years. ESS was least for the age group 60 - 69 years (10.8\%). Gender-wise distribution of OSA based on the ESS was seen to be highest among males $(14.2 \%)$ and females showed a prevalence of $11.7 \%$.

\section{CONCLUSION}

This study is the first epidemiological survey done in Odisha population identifying a higher prevalence of OSA with age. OSA was found to be highly prevalent among males in comparison to females.

\section{KEY WORDS}

Cross-Sectional Studies, Obstructive Sleep Apnoea, Prevalence, Snoring, Surveys and Questionnaires.

HOW TO CITE THIS ARTICLE: Pattanaik S, Rajagopal R, Mohanty N, et al. Prevalence of obstructive sleep apnoea in Odisha population- a camp based questionnaire survey. J. Evolution Med. Dent. Sci. 2018;7(22):2664-2667, D0I: $10.14260 /$ jemds/2018/599

\section{BACKGROUND}

Obstruction of pharynx during sleep along with manifestation of arterial oxygen desaturation, sleep fragmentation and excessive sleepiness during daytime is known as obstructive sleep apnoea. It has been alarmingly increasing globally representing a major public health hazard.

'Financial or Other Competing Interest': None.

Submission 17-04-2018, Peer Review 11-05-2018,

Acceptance 17-05-2018, Published 28-05-2018.

Corresponding Author:

Dr. Snigdha Pattanaik,

Associate Professor,

Department of Orthodontics and Dentofacial Orthopaedics,

Institute of Dental Sciences, Siksha 'O' Anusandhan

(Deemed to be University), Bhubaneswar, Odisha, India.

E-mail:dr.snigdhapattanaik@gmail.com

DOI: $10.14260 /$ jemds $/ 2018 / 599$

However, OSA still remains undetected and is therefore not treated leading to severe morbidity and noticeable mortality. Over the last two decades, OSA has become highly prevalent. ${ }^{1}$ A research done by Peppard et al $^{2}$ using data from Wisconsin Sleep Cohort Study showed prevalence of 10-17\% men and 3-9\% women of moderate or severe OSA.

The significant social and economic impact of excessive day-time sleepiness makes sleep assessment a primary medical need. However, the best screening tool is still a context of debate. The choice of method of screening depends on its capabilities to accomplish certain objectives for inclusion of OSA patients for proper sleep-testing, identify patients with severe condition and exclude patients without disease or those whose evaluation for treatment is less urgent. ${ }^{3}$ 
Various clinical prediction models- Sleep Apnoea Clinical Score (SACS) and Berlin Questionnaire (BQ) have been put forward to prioritise severity of cases by screening.

Among the available questionnaires, The Epworth Sleepiness Scale is a validated tool to assess the daytime sleepiness and can be used for the screening of the OSA.4

There is not much data on the prevalence of OSA in the Indian population, and hence this study was conducted to estimate the prevalence of OSA in Odisha population using Epworth Sleepiness Scale.

\section{MATERIALS AND METHODS}

To collect the data, the questionnaires were distributed in various camps conducted by the Department of Public Health Dentistry. They were explained about obstructive sleep apnoea and its effect on health and were asked to participate in the study. Interested participants were explained about the questionnaire survey in detail and were asked to fill the questionnaire. Finally, the data was collected from 507 male and 505 female participants who were willing to be a part of the survey. The patients were asked to fill the Epworth Sleepiness Scale. The patients were of more than 18 years and did not have any history of major illness. The patients having any history of myocardial infarction, COPD and upperairway surgery were excluded from the study. The subjects were given questionnaires (Epworth Sleepiness Scale) by trained interviewers and were explained regarding it.

\section{Sample Size and Sampling Technique}

The formula for the sample size with (1- $\alpha) \%$ confidence level and with maximum marginal error of estimate of ' $d$ ' for constructing confidence interval of true prevalence using normal approximation has been used. The formula is spelt out as follows:

$\mathrm{N}($ Minimum Sample Size $)=\frac{z_{1-\alpha / 2}^{2} P(1-p)}{d^{2}}$

Where, $\mathrm{P}$ is predetermined value of prevalence of OSA that is ascertained by previous published data that is taken as $30 \%$.

$\alpha=$ the probability of rejecting the null hypothesis, when it is true is taken as $0.05, z_{1-\alpha / 2}^{2}=$ Standard normal value of $\mathrm{Z}$ is taken as 1.96.

$\mathrm{P}=30 \%, \mathrm{~d}=4 \%$ point in absolute term. With this input the minimum sample size ' $n$ ' works out to be 505 .

Since we intended to estimate the same among males and females, our minimum sample size was 505 males and 505 females. The total sample size should be 1010 .

The ESS is an 8-question self-administered questionnaire. Subjects were asked to rate their chances of feeling sleepy while being engaged in eight different activities on a 4-point scale $(0-3)$. Activities enlisted are such chosen that most people will be engaging in those activities at least occasionally if not necessarily every day. The ESS score (the sum of 8 item scores, 0 - 3) ranged from 0 to 24. The higher score indicated that person's average sleep propensity in daily life (ASP) or their 'daytime sleepiness.' It hardly took 23 minutes to complete the questionnaire.

All ESS item-scores are scored in integers $(0$ - 3). If obtained total ESS score includes a half, it was rounded up to the next whole number. If one or more item-scores are missing, that ESS becomes invalid because it is not feasible to interpret missing item-scores. The ESS score (the sum of 8 item-scores) is the only number required under most circumstances. ${ }^{5}$

The questions comprised of presence of snoring, its intensity, the presence of choking during sleep, recurrent awakenings from sleep, prior medical history, medication use, alcohol consumption and smoking history. Snoring more than 5 days/ week is regarded as Habitual Snoring. Daytime hypersomnolence (DHS) was defined as sleepiness at least 3 or more days/ week during the past 3 months in one or more of the following: after awakening, during free time, at work or driving, or during daytime in general. Physical examination was performed- height, weight, neck, waist and hip girth, and blood pressure were measured. The subjects were considered hypertensive if they were currently receiving antihypertensive medication or if their systolic blood pressure was $160 \mathrm{mmHg}$ or more or diastolic blood pressure were $95 \mathrm{mmHg}$ or more. All questionnaire respondents were informed and elaborately explained about this study in a faceto-face interview and were informed that they would be contacted later.

\section{Statistical Analysis}

SPSS version 16.0 software was used to analyse the data collected through questionnaires. Descriptive statistics were done based on the demographic data. The association of age and gender with ESS was done using Chi-square test. Results were presented with $95 \%$ confidence interval.

\section{RESULTS}

A total sample of 1012 subjects who reported to the hospital for a routine check-up were included in the study, of which 508 were males and 504 were females. From these samples, 424 were in the age group of 18 - 29 years, 166 were in the age group of 30 - 39 years, 183 were in the age group of 40 49 years, 124 were in the age group of 50 - 59 years, 83 were in the age group of $60-69$ years and 32 were aged above 70 years.

The ESS was seen to be highest in the age group above 70 (18.8\%) followed by age group 50 - 59 (17.7\%). ESS for the age group 40 - 49 years was $13 \%$ and $11.4 \%$ for the age group 40 - 49 years. ESS was least for the age group 60 - 69 years $(10.8 \%)$. This might be due to less number of subjects in that age group. However, there was no significant association of prevalence of OSA with the age $(p=0.44)$.

Gender-wise distribution of OSA based on the ESS was seen to be highest among males (14.2\%) and females showed $11.7 \%$. However, there was no statistical correlation of gender with prevalence of OSA $(p=0.24)$.

\section{DISCUSSION}

Obstructive sleep apnoea has emerged as a relatively common condition occurring due to recurrent obstruction of upper airway. Excessive daytime sleepiness appears as secondary but usual complaint of the patients, which may further aggravate and indirectly cause complications like systemic hypertension, right heart failure and cardiac arrhythmias.

Lowe et al $^{6}$ described about the development of negative intrapharyngeal during inspiration and at this period collapse 
of airway is prevented by the action of the pharyngeal abductor and dilator muscles.

Rhythmic activation of these muscles occurs during daytime respiration, but like skeletal muscles the hypotonicity of muscles occur during sleep and thus airway stability becomes dependent on pharyngeal size and pharyngeal tissue compliance. This leads to reduction in dimension of airway leading to OSA. OSA is more prevalent in men (2 - 3 times) in comparison to women and this could be attributed to the pattern of fat distribution and difference in sex hormones.

Obesity is the leading predisposing factor for OSA. Centripetal pattern of obesity is linked to peripheral pattern, because fat is preferentially distributed in the abdominal viscera, upper body and neck which causes narrowing of the pharyngeal lumen, thereby obstructing the upper airway leading to OSA. Obese women are less susceptible to the development of OSA than obese men, most likely because of women mostly have peripheral pattern of fat deposition. ${ }^{7}$

OSA is associated with various chronic effects and may lead to hazardous co-morbidities if left undiagnosed. It is characterised by markedly elevated sympathetic nerve traffic while awake, which may promote chronic elevation of blood pressure. Increased left ventricular wall thickening has been observed in OSA. ${ }^{8}$

In a survey done by Wang Zhang and Liang-Yi $\mathrm{Si},{ }^{9}$ it was found that left ventricular hypertrophy is an adverse prognosis of OSA and may also lead to impaired myocardial contractility.

Studies have suggested racial differences in factors contributing to the pathogenesis of OSA. Palmer et al ${ }^{10}$ noticed in comparison to Caucasians, Asians have shorter maxillae and mandible, smaller anterior-posterior facial dimensions and lower BMI. This suggests that constriction of the bony cage is of greater importance than obesity and soft tissue factors in the pathogenesis of OSA in Asians.

If left undiagnosed Obstructive sleep apnoea (OSA) may lead to life-threatening consequences. Polysomnography remains "gold standard" for OSA diagnosis; however, it is of high cost and less accessibility. Thus, various questionnaire methods have been developed to screen OSA like American Society of Anesthesiologists (ASA), STOP, STOP-BANG, Wisconsin, Berlin Questionnaire and Epworth Sleepiness Scale-

- $\quad$ STOP questionnaire (snoring, tiredness, observed apnoea and high blood pressure).

- $\quad$ STOP- Bang questionnaire (STOP including BMI, age, neck circumference, gender) were validated on surgical patients.

- The Wisconsin questionnaire, which was validated on patients selected from the general population.

- Berlin questionnaire was validated in different target populations, i.e. sleep clinic patients, general population and surgical patients.

- Epworth Sleepiness Scale (ESS) is a standard questionnaire to measure daytime sleepiness.

Various studies have been conducted for assessing the prevalence in different ethnic and racial groups; however, most of these studies were limited to white population. Hence, the global prevalence of OSA cannot be reliably compared. Ip and his colleagues ${ }^{11}$ studied on a group of
Chinese population and found that there was $4 \%$ of similarity with the Western studies. Another study done by Li et al ${ }^{12}$ showed that East-Asian men were less obese, but had a greater severity of OSA. Udwadia et $\mathrm{al}^{13}$ evaluated and confirmed the prevalence of OSA in the urban population. It was $7.5 \%$ higher than the rates obtained from epidemiological studies across the globe. This is the first population-based survey of prevalence of OSA done in Odisha.

In the present study, the prevalence of OSA could be clearly related to increase of age. OSA was more prevalent in the older age group ( $>70$ years) $18.8 \%$. It was similar to a study done by Redline and co-workers, ${ }^{14}$ which concluded that the prevalence rates increased five-fold times for the older group. This suggested a strong co-relation between AHI and age. However, another study done by Udwadia et al ${ }^{13}$ had concluded in their study that age was not a significant factor relating to occurrence of OSA. There was highest prevalence of OSA in the age group 45 - 54 yrs. This clearly indicates that age being a strong risk-factor for sleep-disorder breathing over the middle decades of life.

The present study also compared gender difference with respect to the prevalence of OSA. The prevalence of OSA was seen to be highest among males (14.2\%) and females showed a prevalence rate of $11.7 \%$. Young and co-workers 15 in their study found OSA was severely prevalent in males (4\%) and females (2\%). Udwadia et al ${ }^{13}$ also found that hypersomnolence was $21 \%$ in males and $26 \%$ in females based on Apnoea-Hypopnoea Index. Thurnheer et al ${ }^{16}$ compared prevalence of OSA in both the genders and accounted body-size to be a reason for upper airway crosssectional area, which was found to be similar in men and women. However, during NREM sleep stage in men with OSA, the UA was found to be more collapsible thus with greater tendency to OSA. Women are however spared from OSA, because of less collapsibility of UA for a given body mass index.

Bixler $^{17}$ et al studied about pre- and post-menopausal women and OSA prevalence. They concluded that OSA prevalence is comparatively higher in postmenopausal than premenopausal women. Progesterone being a respiratory stimulant may be attributed to the stabilisation of respiratory control system protecting against OSA. On the contrary, fat deposition in the neck is influenced by testosterone leading to the development of OSA through reduction in UA size.

\section{CONCLUSION}

Within the limitations of the present study, it can be concluded that prevalence of OSA is considerably high with noticeable variation and increase in age and gender. Habitual snoring and cessation of breathing during sleep was found to be highly associated with OSA. Males are more commonly affected by OSA compared to females.

\section{REFERENCES}

[1] Lowe AA, Santamaria JD, Fleetham JA, et al. Facial morphology and Obstructive sleep apnea. Am J Orthod Dentofacial Orthop 1986;90(6):484-91.

[2] Peppard PE, Young T, Barnet JH, et al. Increased prevalence of sleep--disordered breathing in adults. Am J Epidemiol 2013;177(9):1006-14. 


\section{Jemds.com}

[3] Ryan CM, Bradley TD. Pathogenesis of obstructive sleep apnea. J Appl Physiol 2005;99(6):2440-50.

[4] Osman EZ, Osborne J, Hill PD, et al. The Epworth Sleepiness Scale: can it be used for sleep apnoea screening among snorers? Clin Otolaryngol Allied Sci 1999;24(3):239-41.

[5] Johns MW. A new method for measuring daytime sleepiness: the Epworth sleepiness scale. Sleep 1991;14(6):540-5.

[6] Akanbi MO, Agaba PA, Ozoh OB, et al. Obesity and obstructive sleep apnea risk among Nigerians. J Med Trop 2017;19(2):110-5.

[7] Palmer LJ, Buxbaum SG, Larkin EK, et al. Whole genome scan for obstructive sleep apnea and obesity in African-American families. Am J Respir Crit Care Med 2004;169(12):1314-21.

[8] Pinto JA, Ribeiro DK, Cavallini AF, et al. Comorbidities associated with obstructive sleep apnea: a retrospective study. Int Arch Otorhinolaryngol 2016;20(2):145-50.

[9] Zhang W, Si LY. Obstructive sleep apnea syndrome (OSAS) and hypertension: pathogenic mechanisms and possible therapeutic approaches. Ups J Med Sci 2012;117(4):370-82.

[10] Palmer LJ, Buxbaum SG, Larkin E, et al. A wholegenome scan for obstructive sleep apnea and obesity. Am J Hum Genet 2003;72(2):340-50.

\section{Original Research Article}

[11] Ip MS, Lam B, Louder IJ, et al. A community study of sleep-disordered breathing in middle-aged Chinese men in Hong Kong. Chest 2001;119(1):62-9.

[12] Li KK, Powell NB, Kushida C, et al. A comparison of Asian and white patients with obstructive sleep apnea syndrome. Laryngoscope 1999;109(12):1937-40.

[13] Udwadia ZF, Doshi AV, Lonkar SG, et al. Prevalence of sleep-disordered breathing and sleep apnea in middleaged urban Indian men. Am J Respir Crit Care Med 2004;169(2):168-73.

[14] Redline S, Tishler P, Avlor J, et al. Prevalence and riskfactors for seep-disordered breathing in children. Am J Respir Crit Care Med 1997;155:A843.

[15] Young T, Palta M, Dempsey J, et al. The occurrence of sleep-disordered breathing among middle-aged adults. N Engl J Med 1993;328(17):1230-5.

[16] Thurnheer R, Wraith PK, Douglas NJ. Influence of age and gender on upper airway resistance in NREM and REM sleep. J Appl Physiol (1985) 2001;90(3):981-8.

[17] Bixler EO, Vgontzas AN, Lin HM, et al. Prevalence of sleep-disordered breathing in women: effects of gender. Am J Respir Crit Care Med 2001;163(3 Pt 1):608-13. 